\title{
Sentiment Intensity Analysis of Informal Texts
}

\author{
Imranul Kabir Chowdhury \\ Executive (Information \\ System) \\ Pedrollo NK Limited
}

\author{
Subhenur Latif \\ Senior Lecturer \\ Department of CSE \\ Daffodil International University
}

\author{
Md. Saddam Hossain \\ Assistant Professor \\ Department of CS \\ American International \\ University- Bangladesh
}

\begin{abstract}
This paper presents a method for an automatic collection of a corpus that can be used to train a sentiment classifier which determines whether an expression is neutral or polar. Depending on the words from the comments of online social networking platform, the human sentiment can be easily extracted, if we can make a machine to understand this extraction by defining some determined hypothesis. The automatic identification leads to enormous application domains for this machine readable sentiment concept. Microblogging web-sites are used here as rich sources of data for opinion mining and sentiment analysis which is tested on well-known training data sets. The results are significantly better than baseline that may suggest people regarding their specific interests based on their respective sentiment studies which can be extended to further business analysis to advice consumer about the negative impact of any issue subjected.
\end{abstract}

\section{Keywords}

NLP, Sentiment Analysis, Opinion Mining, Machine learning

\section{INTRODUCTION}

There are enormous social platforms for networking between friends' colleagues and family members and many other groups. Facebook, twitter, Google plus[1][2][3] are the popular one among them. Millions of users share sentiments on diverse aspects of life daily. Hence micro blogging websites are rich sources of data for various cognitive expressions: opinions, interests, expressions, feelings and sentiment analysis. By using these personal parameters we can identify the peoples' sentiment.

Sentiment analysis aims to identify and extract opinions and attitudes from a given piece of text towards a specific subject[4]. There has been much progress on sentiment analysis of conventional text, which is usually found in open forums, blogs and the typical review channels. However, sentiment analysis of micro blogs is considered as a much harder problem due the unique characteristics possessed by micro blogs (e.g. short length of status updates and language variations)

We use micro blogging and more particularly Facebook for the following reasons:

- Micro blogging platforms are used by diverse group of individuals to express their belief about different issues; therefore it is a valued source of individual's opinions.

- Facebook comprises huge amount of text posts and it grows day by day.

- Facebook's user varies from regular users to celebrities, company representative.
- According to a January 2010 article on InsideFacebook.com, users spent nearly 7 hours per person on Facebook in December 2009, far higher than the other top 10 parent companies on the Internet.

Business firms are interested in some issues, like- What is the public thought regarding their products? Are the people thinking positive or negative about change in product or service?

Political parties may be interested to know if people support their approach or not. All this information can be obtained from microblogging services, as their users post everyday what they like/dislike, and their opinions on many aspects of their life.

Applied part of our analysis may include the following functionalities:

- Understanding user sentiment, to relate a topic of interest for effective ad-targeting.

- If users are biased to any party or organization, topics related to their preference may be presented upon them.

- Users of specific mindset can be connected with similar community. For example - users use mostly playful status may be presented with local comedy club information.

First, we will categorize this sentiment in three types: positive sentiment, negative sentiment and neutral sentiment[5]. Thereby we can recognize this concept using polarity[5]. Here our main concern is to analysis the public comments of one's by giving most generalized form of hypothesis. There are very few works by the researchers based on this polarity. The main challenge of this type of work is - public comments do not follow the lexicon pattern. We have to depend on one's acculturation, local accent, socialization. We will not pertain more dimensions into these factors rather we prefer more generalized ones. Our work will depend on a defined target that is positive or negative polarity. We will show how to build a domain-independent sentiment classifier learned from short textual sparse data, which is able to operates in a streaming fashion, and adapt dynamically to the new data. In this paper we emphasized on the polarity of sentiment bearing expression. Here we will propose an algorithm which measure the percentage of polarity of the positive and negative polarity and gives the sentiment result of ones. We will also give a structure of sentiment result based on the human emotion. Our approach will be based on the following assumption:

At the outset it will find the root word in the corpus and then it will define the polarity, consequently it will search for the next root word and find the consistency with the previous polarity. Based on the consistency and inconsistency an inter- 
relationship network will be developed to find the resultant goal.

\section{RELATED WORKS}

The emergence of social media combined with micro blogging services' easy-to-use features have dramatically changed people's life with more and more people sharing their thoughts, expressing opinions, and seeking for support on such open social and highly connected environments.

Monitoring and analyzing opinions from social media provides enormous opportunities for both public and private sectors. For private sectors, it has been observed [6][7] that the reputation of a certain product or company is highly affected by rumors and negative opinions published and shared among users on social networks. Understanding this observation, companies realize that monitoring and detecting public opinions from micro blogs leading to building better relationships with their customers, better understanding of their customers' needs and better response to changes in the market.

Open nature of micro blogs poses an open-domain problem where classifiers should work in a multi-domain environment.

SAS[8] sentiment Analysis automatically rates and classifies opinions expressed in electronic text. It collects text inputs from websites, social media outlets and internal file systems, and then puts them in a unified format to assess relevance to predefined topics. The reports identify trends or emotional changes, and an interactive workbench allows subject-matter experts to refine sentiment models. The solution automatically scores input documents as they're received, providing realtime updates about sentiment changes. By assessing sentiment in real time, the software provides quantified insights into the overall impressions people have of products, services and brands.

We can conclude that the lack of labeled data needed for classifier training.For public sectors, recent studies[9][10] show that there is a strong correlation between activities on social networks and the outcomes of certain political issues. For example, Twitter and Facebook were used to organize demonstrations and build solidarity during Arab Spring of civil uprising in Egypt, Tunisia, and currently in Syria. One week before Egyptian president's resignation the total rate of tweets about political change in Egypt increased ten-fold. In Syria, the amount of online content produced by opposition groups in Facebook increased dramatically. Another example is the UK General Election 2010. It has been shown that activities on Twitter are a good predicator of popularities of political parties[11].

Thus tracking and analyzing users' activities on social media are the key to understanding and predicting public opinions towards certain political event.

Anthony brew et al. described such a system[12] based on Twitter that maintains a happiness index for nine US cities. The main contribution of this paper is a companion system called Sentire Crowds that allows us to identify the underlying causes behind shifts in sentiment. This ability to analyze the components of the sentiment signal highlights a number of problems. It shows that sentiment scoring on social media data without considering context is difficult. More importantly, it highlights cases where sentiment scoring methods are susceptible to unexpected shifts due to noise and trending memes.

The short length of status updates coupled with their noisy nature makes the data very sparse to analyze using standard machine learning classifiers.

Mike et al. [13] reports a study of a month of English Twitter posts, assessing whether popular events are typically associated with increases in sentiment strength, as seems intuitively likely. Their results give strong evidence that popular events are normally associated with increases in negative sentiment strength and some evidence that peaks of interest in events have stronger positive sentiment than the time before the peak. It seems that many positive events, such as the Oscars, are capable of generating increased negative sentiment in reaction to them. Nevertheless, the surprisingly small average change in sentiment associated with popular events is consistent with events affording posters opportunities to satisfy pre-existing personal goals more often than eliciting instinctive reactions.

The streaming fashion of microblogs where data arrives at a high speed. This means data should be processed in real time and classifiers should adapt quickly with the newly arrived data.

\section{SYSTEM DESCRIPTION}

System description is an essential issue regarding Natural language Processing. After reviewing lots of papers related to our dissertation we have come up with some new innovative features along with the existing approach. We have tried to give a very generalized and specific description rather than the conventional one. We delineated our views in a different way for empathy of every level of technicalities subjected. All the system regards subjected have been introduced our system as followings:

\section{High level Description \\ 2. Low level Description.}

\subsection{High Level Description}

This is our high level description so that anyone can understand the steps from the top level irrespective to lower level details. In this level our system interpret few steps as shown in Fig. 1. After collecting the corpus we spilt the texts in to sentences then we assign the polarity of each sentences and check the spell algorithm whether there exists any repeated words. Collectively we named these three steps as Data manipulation. Sentiment extraction process basically calculate the sentiment of one's based on their Facebook comments at most. Details are described in the next section (low level description). Accuracy factors are introduced to find the preciseness in sentiment extraction. Finally the result comes out after performed by all steps. 


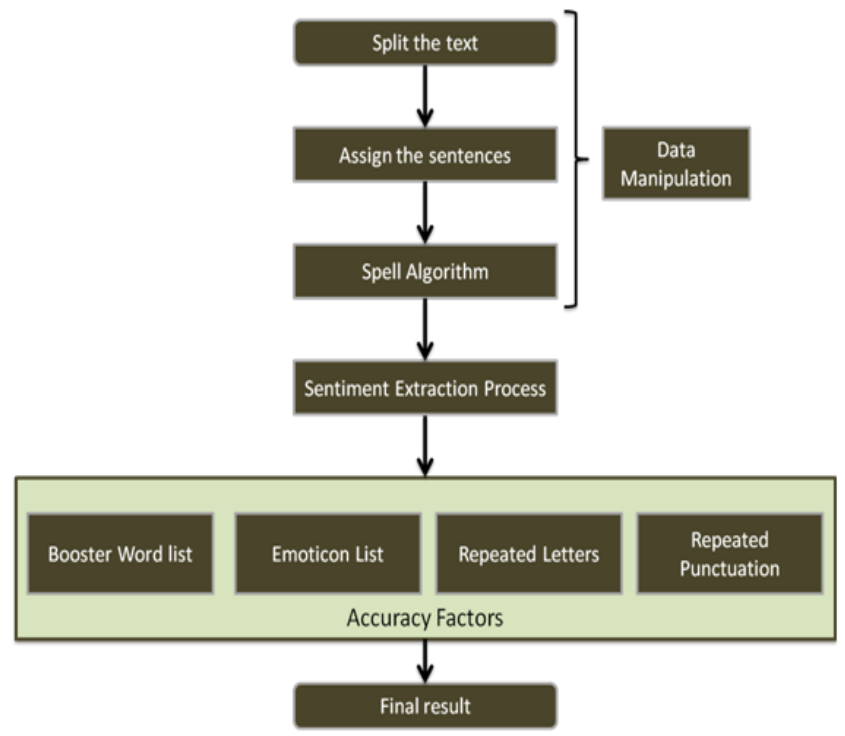

Figure 1: High Level Description

\subsection{Low-level Description}

This low level description includes detailed depiction of all the high level steps subjected. Figure 2 represents our worth internal system.

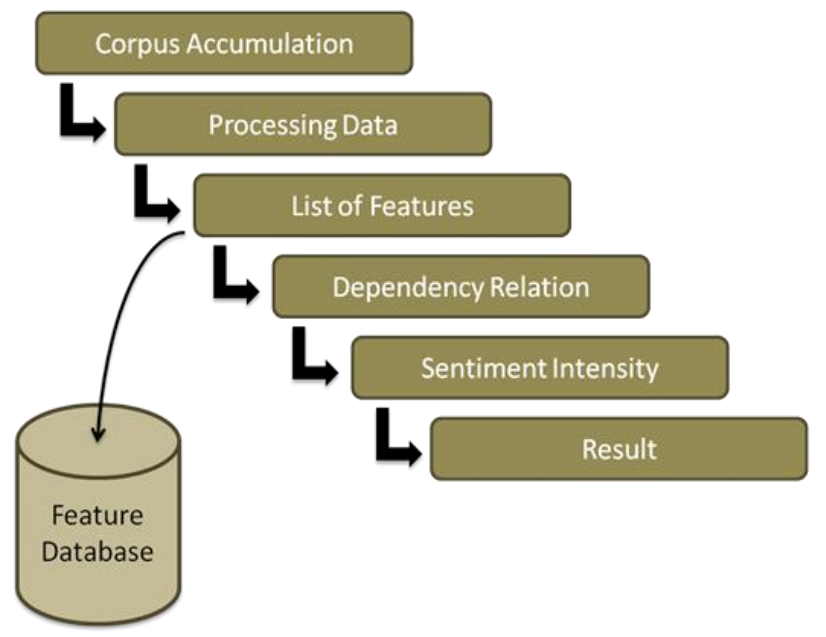

Figure 2: Low Level Description

\section{Corpus Accumulation}

Using Facebook API we collected a corpus of text posts and formed a dataset of three classes: positive sentiments, negative sentiments, and a set of objective texts (no sentiments). To collect negative and positive sentiments, we followed the same procedure as in [14][15]. We queried Facebook for two types of emoticons:

• Happy emoticons: “:-)”, “:)”, “=)”, “:D” etc.
• Sad emoticons: “:-(", “:(", “=(", “;(" etc.

The two types of collecting corpora will be used to train a classifier to recognize positive and negative sentiments. In order to collect a corpus of objective posts, we retrieved text messages from Facebook accounts of popular newspapers and magazines , such as "bd news 24"[16], "Bangla news"[17] etc. We queried accounts of 8 newspapers to collect a training set of objective texts. Because each message cannot exceed 140 characters by the rules of the microblogging platform, it is usually composed of a single sentence. Therefore, we assume that an emoticon within a message represents an emotion for the whole message and all the words of the message are related to this emotion. In our research, we use English language.

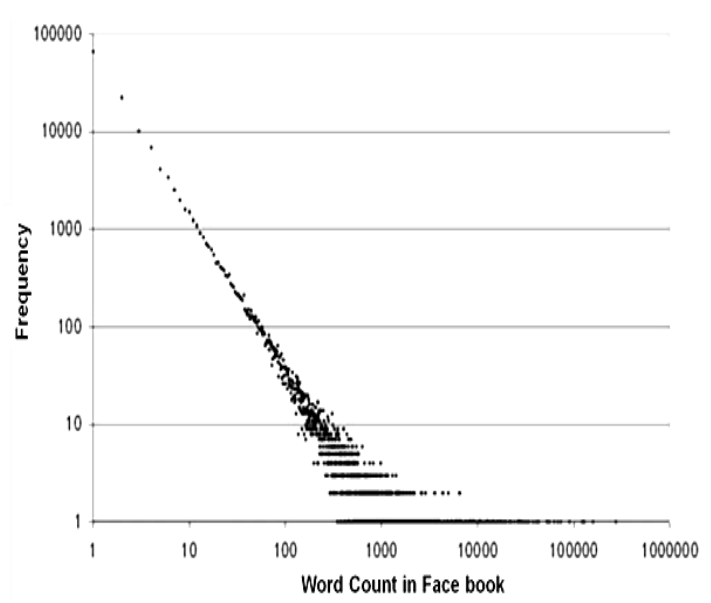

Figure 3: The Distribution of word count follows Zipf's law

\section{Processing Data}

First, we checked the distribution of words frequencies in the corpus. A plot of word frequencies is presented in above Figure 3. As we can see from the plot, the distribution of word frequencies follows Zipf's law[18], which confirms a proper characteristic of the collected corpus. Next, we used TreeTagger [19] for English to tag all the posts in the corpus. We are interested in a difference of tags distributions between sets of texts (positive, negative, and neutral). To perform a pair wise comparison of tags distributions, we calculated the following values for each tag and two sets (i.e. positive and negative posts):

$$
P_{1,2}^{T}=\frac{N_{1}^{T}-N_{2}^{T}}{N_{1}^{T}+N_{2}^{T}}
$$

Where $N_{1}^{T}$ and $N_{2}^{T}$ are numbers of tag $\mathrm{T}$ occurrences in the first and second sets respectively. 


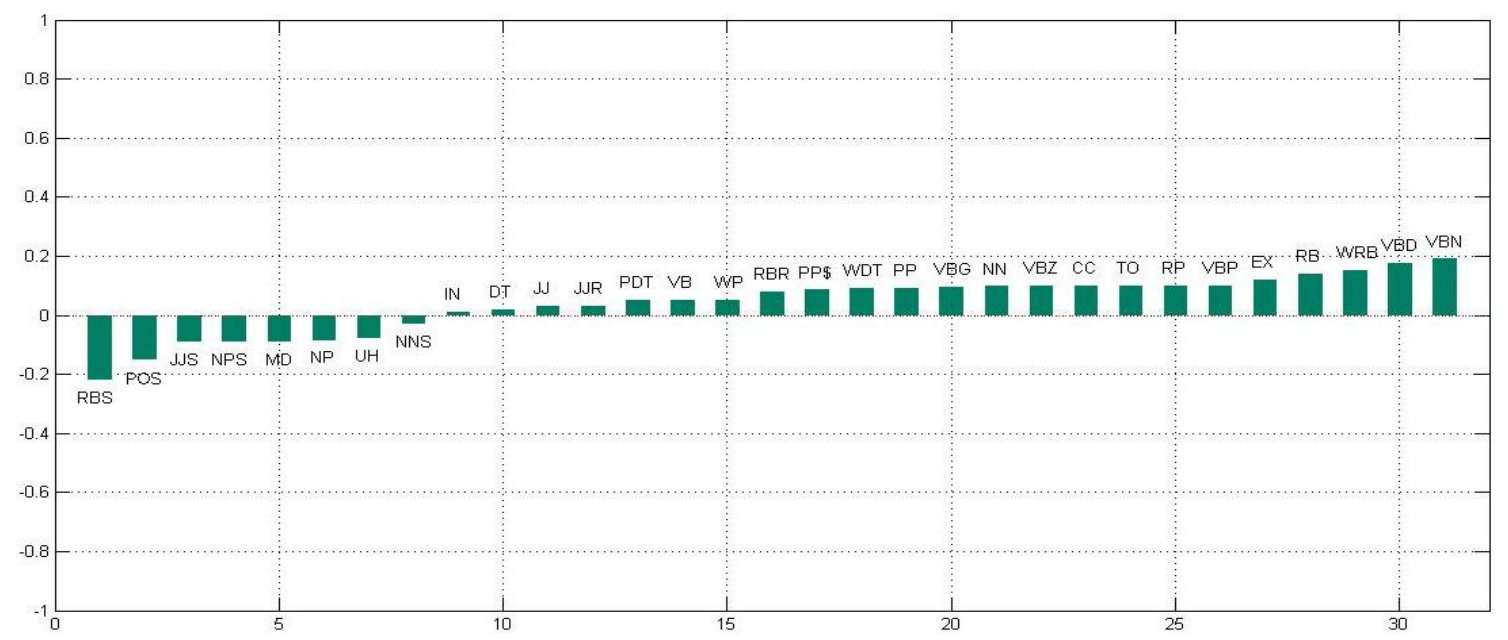

Figure 4: $\mathrm{P}^{\mathrm{T}}$ values for Positive vs. Negative

\section{Polarity vs. Neutral Sets}

Figure 4 shows the values of $\mathrm{P}^{\mathrm{T}}$ across all the tags where set 1 is a polarity set (mixture of the positive and the negative sets) and set 2 is a neutral set (the neutral set). From the graph we can observe that POS tags are not distributed evenly in two sets, and therefore can be used as indicators of a set. For example, utterances (UH) can be a strong indicator of a polarity text. Next, we will explain the observed phenomena.

We can observe that neutral texts tend to contain more common and proper nouns (NPS, NP, NNS), while authors of polarity texts use more often personal pronouns (PP, PP\$). Authors of subjective texts usually describe themselves (first person) or address the audience (second person) (VBP), while verbs in polarity texts are usually in the third person (VBZ). As for the tense, polarity texts tend to use simple past tense (VBD) instead of the past participle (VBN). Also a base form of verbs (VB) is used often in polarity texts, which is explained by the frequent use of modal verbs (MD). In the graph, we see that superlative adjectives (JJS) are used more often for expressing emotions and opinions, and comparative adjectives (JJR) are used for stating facts and providing information. Adverbs (RB) are mostly used in polarity texts to give an emotional color to a verb. Figure 5 shows the values of $\mathrm{P}^{\mathrm{T}}$ for negative and positive sets. Another indicator of a positive text is superlative adverbs (RBS), such as "most" and "best". Positive texts are also characterized by the use of possessive ending (POS). As opposite to the positive set, the negative set contains more often verbs in the past tense (VBN, VBD), because many authors express their negative sentiments about their loss or disappointment. Here is an example of the most frequent verbs:

"missed", "bored", "gone", "lost", "stuck", "taken". We have compared distributions of POS-tags in two parts of the same sets (e.g. a half of the positive set with another half of the positive set). The proximity of the obtained distributions allows us to conclude on the homogeneity of the corpus.

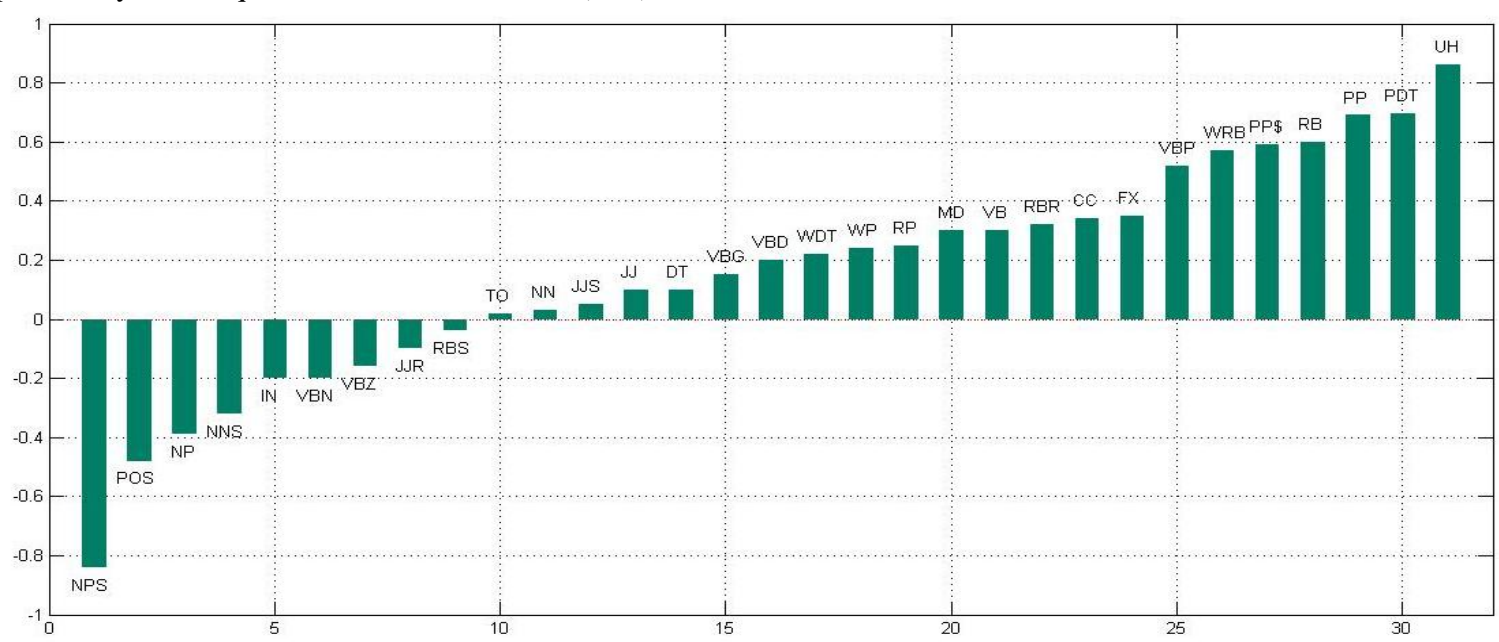

\section{List of Features}

The collected dataset is used to extract features that will be used to train our sentiment classifier. We used the presence of an n-gram as a binary feature, while for general information retrieval purposes, the frequency of a keyword's occurrence is a more suitable feature, since the overall sentiment may not necessarily be indicated through the repeated use of keywords. Pang et al. have obtained better results by using a

\section{Polarity Sets}

term presence rather than its frequency [20]. We have experimented with unigrams, bigrams, and trigrams. Pang et al. [20] reported that unigrams outperform bigrams when performing the sentiment classification of movie reviews, and Dave et al. [21] have obtained contrary results: bigrams and trigrams worked better for the product-review polarity classification. 
We tried to determine the best settings for the microblogging data. On one hand high-order n-grams, such as trigrams, should better capture patterns of sentiments expressions.

$$
P(s \mid M) \sim P(G \mid s) \cdot P(T \mid S)
$$

On the other hand, unigrams should provide a good coverage of the data. The process of obtaining n-grams from a facebook post is as follows:

$$
P(G \mid s)=\prod_{g \in G} P(g \mid s)
$$

1. Filtering - we remove URL links (e.g. http://example.com), facebook user names (e.g. @imran - with symbol @ indicating a user name).

2. Tokenization - we segment text by splitting it by spaces and punctuation marks, and form a bag of words. However, we make sure that short forms such as "don't", "I'll", "she'd" will remain as one word.

3. Removing stopwords - we remove articles ("a", "an", "the") from the bag of words.

4. Constructing n-grams - we make a set of n-grams out of consecutive words.

A negation (such as "no" and "not") is attached to a word which precedes it or follows it. For example, a sentence "I do not like fish" will form two bigrams: "I do+not", "do+not like", "not+like fish". Such a procedure allows to improve the accuracy of the classification since the negation plays a special role in an opinion and sentiment expression [22]. This idea of above process follows the same concept described in [23].

\section{Dependency Relation}

We build a sentiment classifier using the multinomial Naive Bayes classifier. We also tried SVM [24] and CRF [25] however the Naive Bayes classifier yielded the best results. Naive Bayes classifier is based on Bayes' theorem [26].

$$
P(s \mid M)=\frac{P(s) \cdot P(M \mid s)}{P(M)}
$$

where $\mathrm{s}$ is a sentiment, $\mathrm{M}$ is a facebook message. Because, we have equal sets of positive, negative and neutral messages, we simplify the equation:

$$
\begin{aligned}
& P(s \mid M)=\frac{P(M \mid s)}{P(M)} \\
& P(s \mid M) \sim P(M \mid s)
\end{aligned}
$$

We train two Bayes classifiers, which use different features: presence of n-grams and part-of-speech distribution information. $\mathrm{N}$-gram based classifier uses the presence of an $\mathrm{n}$-gram in the post as a binary feature.

The classifier based on POS distribution estimates probability of POS-tags presence within different sets of texts and uses it to calculate posterior probability. Although, POS is dependent on the n-grams, we make an assumption of conditional independence of n-gram features and POS information for the calculation simplicity:

$$
P(s \mid M) \sim P(G \mid s) \cdot P(T \mid S)
$$

Where $\mathrm{G}$ is a set of $\mathrm{n}$-grams representing the message, $\mathrm{T}$ is a set of POS-tags of the message. We assume that n-grams are conditionally independent:

$$
P(G \mid s)=\prod_{g \in G} P(g \mid s)
$$

Similarly, we assume that POS-tags are conditionally independent:

$$
\begin{gathered}
P(T \mid s)=\prod_{t \in G} P(t \mid s) \\
P(s \mid M) \sim \prod_{g \in G} P(g \mid s) \cdot \prod_{t \in G} P(t \mid s)
\end{gathered}
$$

Finally, we calculate log-likelihood of each sentiment:

$$
L(s \mid M)=\sum_{g \in G} \log (P(g \mid s))+\sum_{t \in G} \log (P(t \mid s))
$$

\section{Sentiment Intensity}

To increase the accuracy of the system, we should discard common $n$-grams, i.e. n-grams that do not strongly indicate any sentiment nor indicate objectivity of a sentence. Such ngrams appear evenly in all datasets. To discriminate common n-grams, we also follow the same strategies defined in [23]. In addition to that here we introduced a feature Booster Wordlist to strengthen the accuracy. A Booster Word List contains words that boost or reduce the emotion of subsequent words, whether positive or negative. Each word increases emotion strength by 1 or 2 (e.g., very, extremely) or decreases it by 1(e.g. some). If any comment of Facebook user finds some booster words then the system gives the entropy and the salient values more precise than the previous. Below are the comparisons.

The high value of the entropy indicates that a distribution of the appearance of an n-gram in different sentiment datasets is close to uniform. Therefore, such an n-gram does not contribute much in the classification.

A low value of the entropy on the contrary indicates that an ngram appears in some of sentiment datasets more often than in others and therefore can highlight a sentiment (or objectivity). Thus, to increase the accuracy of the sentiment classification, we would like to use only n-grams with low entropy values. We can control the accuracy by putting a threshold value $\eta$, filtering out $n$-grams with entropy above $\eta$. In regard to the salience it takes a value between 0 and 1 . The low value indicates a low salience of the n-gram, and such an n-gram should be discriminated. Same as with the entropy, we can control the performance of the system by tuning the threshold value $\Omega$. In Table 5.3. examples of $n$-grams with low entropy values and high salience values are presented. Using the entropy and salience, we obtain the final equation of a sentiment's log-alike:

$$
\begin{aligned}
L(s \mid M) & =\sum_{g \in G} \log (P(g \mid s)) \cdot i f(f(g)>\theta, 1,0) \\
& +\sum_{t \in G} \log (P(t \mid s))
\end{aligned}
$$

We have tested our classifier on a set of real Facebook posts hand-annotated. We used the same evaluation set as in [15]. 
The characteristics of the dataset are presented in following Table 1.

Table 1: The characteristics of the evaluation dataset

\begin{tabular}{|l|r|}
\hline Sentiment & Number of Samples \\
\hline Positive & 196 \\
\hline Negative & 80 \\
\hline Neutral & 26 \\
\hline Total & 302 \\
\hline
\end{tabular}

We compute accuracy [27] of the classifier on the whole evaluation dataset, i.e.:

$$
\text { accuracy }=\frac{N(\text { correct } \text { classifications })}{N(\text { all classifications })}
$$

We measure the accuracy across the classifier's decision [28]:

$$
\text { decision }=\frac{N(\text { retrieved documents })}{N(\text { all documents })}
$$

\section{RESULT \& DISCUSSIONS}

First, we have tested the impact of an n-gram order on the classifier's performance. The results of this comparison are presented in Figure 6. As we see from the graph, the best performance is achieved when using bigrams. We explain it as bigrams provide a good balance between a coverage (unigrams) and an ability to capture the sentiment expression patterns (trigrams). Next, we examine the impact of attaching negation words when forming $\mathrm{n}$-grams. The results are presented in Figure 7. From the both figures, we see that we can obtain a very high accuracy, although with a low decision value (11). By adding the booster word list we gain accuracy Thus, if we use our classifier for the sentiment search engine, the outputted results will be very accurate. We have also examined the impact of the dataset size on the performance of the system. To measure the performance, we use Fmeasure[27]:

$F=\left(1+\beta^{2}\right) \frac{\text { precision } \cdot \text { recall }}{\beta^{2} \cdot \text { recall }+ \text { recall }}$

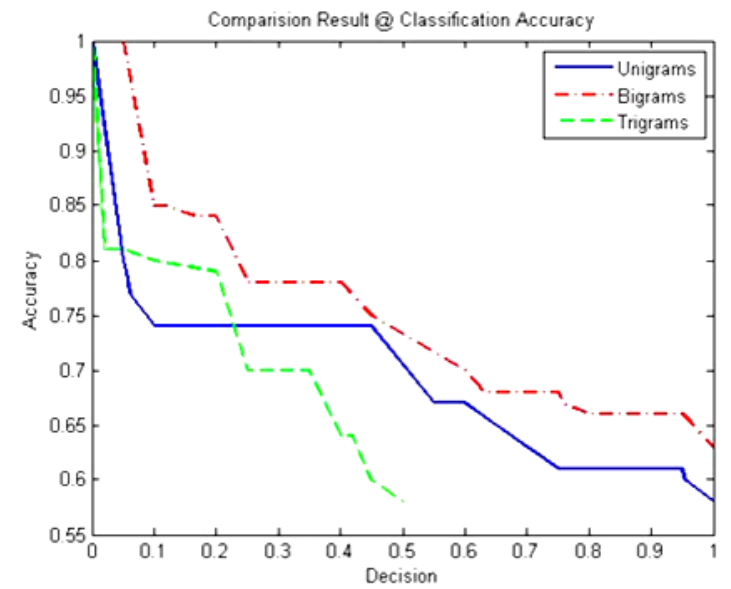

Figure 6: The comparision of the classification accuracy when using unigrams, bigrams, trigrams
In our evaluations, we replace precision with accuracy (12) and recall with decision (11), because we deal with multiple classes rather than binary classification:

$$
F=\left(1+\beta^{2}\right) \frac{\text { accuracy } \cdot \text { decision }}{\beta^{2} \cdot \text { accuracy }+ \text { decision }}
$$

Where $\beta=0.5$. We do not use any filtering of $n$-grams in this experiment. The result is presented on Figure 8. As we see from the graph, by increasing the sample size, we improve the performance of the system. However, at a certain point when the dataset is large enough, the improvement may not be achieved by only increasing the size of the training data. We examined two strategies of filtering out the common n-grams: salience (11) and entropy (10). Figure 9 shows that using the salience provides a better accuracy, therefore the salience discriminates common n-grams better than the entropy.

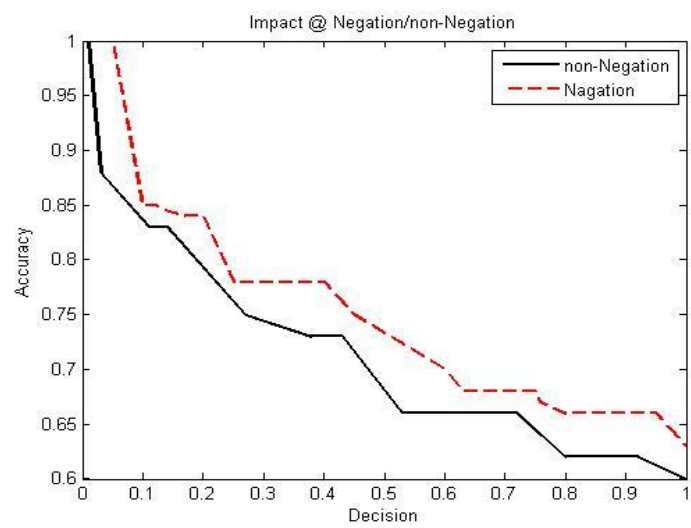

Figure 7: The impact of using the attachment of negation

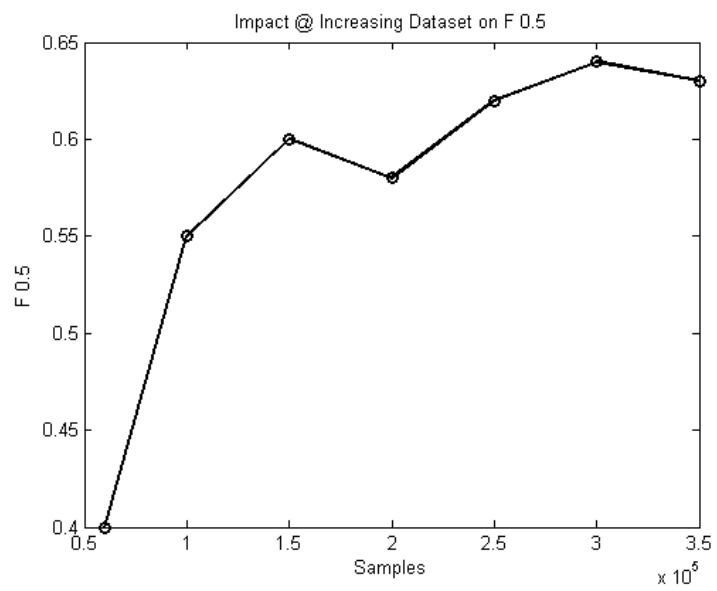

Figure 8: The impact of increasing the dataset size on the F 0.5 measure 


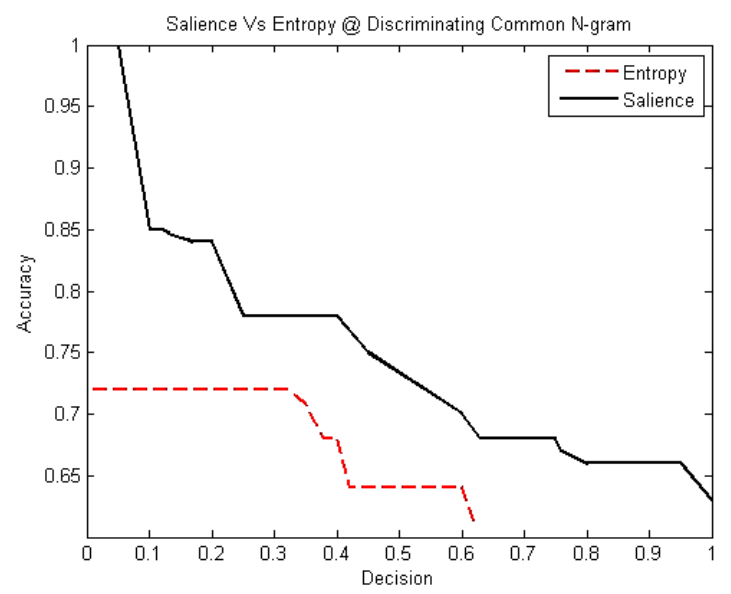

Figure 9: Salience vs. entropy for discriminating common n-grams

\section{CONCLUSION}

Microblogging has nowadays become one of the major types of the communication. A recent dissertation has identified it as online word-of-mouth branding [29]. The huge amount of information contained in microblogging websites makes them an attractive source of data for opinion mining and sentiment analysis. In our research, we have presented a method for an automatic collection of a corpus that can be used to train a sentiment classifier. We consider TreeTagger for POS-tagging and observed the difference in distributions among positive, negative and neutral sets. We have also introduced Booster Word List intensity for more precise on the result of sentiment expression. From the observations we conclude that authors used syntactic structures to describe emotions. Some POStags may be strong indicators of emotional text. We used the collected corpus to train a sentiment classifier. Our classifier is able to determine positive, negative and neutral sentiments of documents and the result is more precise till date. The classifier is based on the multinomial Naive Bayes classifier that uses N-gram and POS-tags as features.

As the future work, we plan to collect a multilingual corpus of Facebook data and compare the characteristics of the corpus across different languages. We plan to use the obtained data to build a multilingual sentiment classifier.

\section{REFERENCES}

[1] "Fcaebook." [Online]. Available: https://www.facebook.com/.

[2] “Twiter." [Online]. Available: https://twitter.com/.

[3] "Google Plus." [Online]. Available: https://www.google.com/intl/en/+/learnmore/better/.

[4] H. Saif, "Sentiment Analysis of Microblogs," no. March, 2012.

[5] L. Chen, W. Wang, M. Nagarajan, S. Wang, and A. P. Sheth, "Extracting Diverse Sentiment Expressions with Target-Dependent Polarity from Twitter," pp. 50-57.

[6] "No WARD, J., AND OSTROM, A. The internet as information minefield:: An analysis of the source and content of brand information yielded by net searches. Journal of Business research 56, 11 (2003), 907-914."

[7] "YOON, E., GUFFEY, H., AND KIJEWSKI, V. The effects of information and company reputation on intentions to buy a business service. Journal of Business Research 27, 3 (1993), 215-228."

[8] F. Sheet, "SAS ® Sentiment Analysis Automatically pinpoint sentiment from the Web and internal electronic documents to understand trends and develop effective strategies."

[9] "BHUIYAN, S. Social media and its effectiveness in the political reform movement in egypt. Middle East Media Educator 1, 1 (2011), 14-20."

[10] "HUSSAIN, M., AND HOWARD, P. the role of digital media. Journal of Democracy 22, 3 (2011), 35-48."

[11] "HE, Y., AND SAIF, H. Quantising Opinons for Political Tweets Analysis. In Proceeding of the The eighth international conference on Language Resources and Evaluation (LREC) - In Submission (2012)."

[12] A. Brew, D. Greene, D. Archambault, and $\mathrm{P}^{\prime}$. Cunningham, "Deriving Insights from National Happiness Indices," 2011 IEEE 11th International Conference on Data Mining Workshops, no. 1, pp. 5360, Dec. 2011.

[13] M. Thelwall, K. Buckley, and G. Paltoglou, "Sentiment in Twitter Events," vol. 62, no. 2, pp. 406-418, 2011.

[14] J. Read, "Using emoticons to reduce dependency in machine learning techniques for sentiment classification," in Proceedings of the ACL Student Research Workshop, 2005, pp. 43-48.

[15] A. Go and L. Huang, "Twitter Sentiment Analysis Introduction Procedure Data Collection," 2009.

[16] "bdnews24.com." [Online]. Available: http://bdnews24.com/. [Accessed: 02-Aug-2013].

[17] "Bangla News 24 - News." [Online]. Available: http://www.banglanews24.com/. [Accessed: 02-Aug2013].

[18] G. K. Zipf, "Selected Studies of the Principle of Relative Frequency in Language.," Cambridge (Mass)., 1932.

[19] S. Helmut, "Probabilistic Part-of-Speech Tagging Using Decision Trees.," 1994.

[20] B. Pang, L. Lee, H. Rd, and S. Jose, "Thumbs up ? Sentiment Classification using Machine Learning Techniques," no. July, pp. 79-86, 2002.

[21] K. Dave, I. Way, S. Lawrence, and D. M. Pennock, "Mining the Peanut Gallery: Opinion Extraction and Semantic Classification of Product Reviews," 2003.

[22] T. Wilson, J. Wiebe, and P. Hoffmann, "Recognizing contextual polarity in phrase-level sentiment analysis," Proceedings of the conference on Human Language Technology and Empirical Methods in Natural Language Processing - HLT '05, pp. 347-354, 2005.

[23] A. Pak and P. Paroubek, "Twitter as a Corpus for Sentiment Analysis and Opinion Mining," pp. 1320 1326.

[24] E. Alpaydın, Introduction to Machine Learning Second Edition. 2004.

[25] J. Lafferty, A. McCallum, and F. Pereira, "Conditional Random Fields: Probabilistic Models for Segmenting and Labeling Sequence Data," 2001. 
International Journal of Computer Applications (0975 - 8887)

Volume 147 - No.10, August 2016

[26] A. Hayter, Probability and statistics for engineers and scientists. Belmont Calif.: Thomson Brooks/Cole ;Duxbury, 2007.

[27] C. D. Manning and H. Schütze, Foundations of statistical natural language processing. Cambridge, MA, USA: MIT Press, 1999.

[28] G. Adda, J. Mariani, J. Lecomte, P. Paroubek, and M. Rajman., "The GRACE French part-of-speech tagging evaluation task.," International Conference on Language Resources and Evaluation, Granada, May., vol. 1, pp. 433-441, 1998.

[29] B. J. Jansen, M. Zhang, K. Sobel, and A. Chowdury, "Micro-blogging as online word of mouth branding.," In proceeding of: Proceedings of the 27th International Conference on Human Factors in Computing Systems, CHI 2009, Extended Abstracts Volume, Boston, MA, USA., 2009. 